\title{
KPP FRONT SPEEDS IN RANDOM SHEARS AND THE PARABOLIC ANDERSON PROBLEM*
}

\author{
JACK XIN ${ }^{\dagger}$
}

\begin{abstract}
We study the asymptotics of front speeds of the reaction-diffusion equations with Kolmogorov-Petrovsky-Piskunov (KPP) nonlinearity and zero mean stationary ergodic Gaussian shear advection on the entire plane. By exploiting connections of KPP front speeds with the almost sure Lyapunov exponents of the parabolic Anderson problem, and with the homogenized Hamiltonians of Hamilton-Jacobi equations, we show that front speeds enhancement is quadratic in the small root mean square (rms) amplitudes of white in time zero mean Gaussian shears, and it grows at the order of the large rms amplitudes. However, front speeds diverge logarithmically if the shears are time independent zero mean stationary ergodic Gaussian fields.
\end{abstract}

1. Introduction. Front propagation in random media appears in many scientific areas, and analysis of prototype models has been an efficient way to improve our understanding of fronts with complex structures [13], [22] etc. We shall consider reaction-diffusion (R-D) fronts moving inside a two dimensional random shear field modeled by:

$$
u_{t}=\frac{1}{2} \triangle_{y} u+\sigma \vec{\xi} \cdot \nabla u+f(u), \quad y=\left(y_{1}, y_{2}\right) \in R^{2}
$$

where $\vec{\xi}=\left(0, \xi\left(y_{1}, t\right)\right), \xi$ a mean zero stationary ergodic Gaussian field, $\sigma>0$ a parameter measuring the shear strength (root mean square amplitude for short), $\triangle_{y}=$ $\partial_{y_{1} y_{1}}^{2}+\partial_{y_{2} y_{2}}^{2}, f(u)=u(1-u)$, known as the KPP nonlinearity. We are interested in fronts moving along the $y_{2}$ direction, and how the speed of propagation depends on the shear field $\vec{\xi}$ at large time.

If the shear $\vec{\xi}$ is deterministic, e.g. periodic in space (and time), the asymptotic behavior of front speed is known for many forms of reaction nonlinearity $f(u)$. For small $\sigma$ and zero mean $\xi$ :

$$
c_{*}=c_{0}\left(1+\alpha \sigma^{2}+h . o . t\right),
$$

where $\alpha$ is a positive constant depending only on $\xi, c_{0}$ is the pure R-D front speed, and h.o.t is short for higher order terms. The constant $\alpha$ is universal among:

- bistable nonlinearity $(f(u)=u(1-u)(u-\mu), \mu \in(0,1 / 2))$;

- ignition combustion nonlinearity $(f(u)=0, u \in(0, \theta), \theta \in(0,1)$, ignition level, $f(u)>0, u \in(\theta, 1))$;

- $\operatorname{KPP}(f(u)=u(1-u))$, and generalizations $\left(f(u)=u^{m}(1-u), m \geq 2\right)$.

See [17], [11] for calculations using perturbation and variational methods in the spatially periodic case for most of the above nonlinearities. The robustness of (1.2) in the spatially-temporally periodic case is studied recently in [15]. Except for the KPP nonlinearity, where linearization is possible to obtain front speeds, one needs to work with an exact traveling wave solution and the resulting equation it satisfies in order to find the constant $\alpha$.

\footnotetext{
*Received December 19, 2002; accepted for publication May 14, 2003.

${ }^{\dagger}$ Department of Mathematics and ICES, University of Texas at Austin, Austin, TX 78712, USA (jxin@mail.ma.utexas.edu).
} 
For large $\sigma$, on the other hand:

$$
c_{*}=O(\sigma)
$$

if $f(u) \geq 0$, see [1], [7], [12], [10], [21], and existence of limit $c_{*} / \sigma$ holds for the KPP [3]. The asymptotics (1.3) also hold in the spatially-temporally periodic shears [15], and is numerically observed even for bistable nonlinearity [15].

A natural question is whether these results remain valid for fronts in random shears. We approach this issue here for the KPP nonlinearity and in the entire space $R^{2}$. We show that the KPP front speed formula is connected with the almost sure Lyapunov exponent of the so called parabolic Anderson problem, namely the exponential growth rate of the semigroup $e^{t L}$ with $L \cdot=\Delta \cdot+V(x, t) \cdot, V(x, t)$ a Gaussian process with mean zero. There is a rich literature on almost sure Lyapunov exponent [4], [5], [6]. The logarithm of the positive solutions of the linear parabolic equation $u_{t}=L u$ satisfies the stochastic viscous KPZ equation in surface growth [2]. It is a quadratic Hamilton-Jacobi equation with random potential and viscosity, and the growth rate of solutions is also the effective Hamiltonian in the large space large time (homogenization) limit [22]. Combining these two connections, we are able to simplify the front speed calculations. The main finding is: if $\xi=\xi\left(y_{1}, t\right)$, white in time, mean zero stationary ergodic Gaussian process, then almost surely front speed in random shears is a deterministic constant $c_{*}$ with asymptotics:

$$
c^{*}=c_{0}\left(1+\frac{1}{2} \Gamma(0) \sigma^{2}+\text { h.o.t }\right), \quad \sigma \ll 1,
$$

$\Gamma(\cdot)$ the spatial covariance function of $\xi, \Gamma(0)>0$; and:

$$
c^{*}=O(\sigma), \quad \sigma \gg 1
$$

However, if $\xi=\xi\left(y_{1}\right)$, time independent mean zero stationary ergodic Gaussian process, then almost surely, the front velocity diverges like:

$$
c^{*} \sim c_{0}+\sigma \sqrt{2 \Gamma(0) \log t}, \quad t \gg 1,
$$

due to the almost sure growth of running maxima of Gaussian process. The divergence can be faster for other types of noisy processes, for example the Poisson shot noise.

Comparing (1.4), (1.5) and (1.6), we see that the fast switching of direction in time helps to slow down fronts, and cures the divergence in (1.6). The slow down of front speeds due to fast direction changing in time also occurs in the deterministic setting for all the above nonlinearities [15]. As the front speed asymptotics in the deterministic cases (1.2)-(1.3) are not sensitive to nonlinearities, we expect that the analysis on random KPP front speeds, (1.4)-(1.6), will have implications for other types of $f(u)$. Numerical work along this line is in progress and will be reported elsewhere [16]. It is interesting to study the regime of non-white time correlated shears.

The rest of the paper is organized as follows. In section 2 , we derive the front speed asymptotic formula (1.4), (1.6), and bound (1.5), using properties of homogenized Hamiltonian and asymptotics of Lyapunov exponents. In section 3, we draw conclusions and discuss future works.

2. KPP Front Speeds in Random Shear Flows. The KPP (minimal) front speeds in random media have a variational characterization ( [9], [22], and references therein), only $f^{\prime}(0)$ enters from nonlinearity in an otherwise linear problem. 
Let $\xi=\xi\left(y_{1}, t, \omega\right)$ be a mean zero stationary spatial-temporal Gaussian field, with covariance function:

$$
E\left(\xi\left(y_{1}, t\right) \xi\left(y_{1}^{\prime}, t^{\prime}\right)\right)=\Gamma\left(\left|t-t^{\prime}\right|,\left|y_{1}-y_{1}^{\prime}\right|\right),
$$

$\forall y, y^{\prime} \in R^{1}, t, t^{\prime} \in R^{1}$, where $\Gamma$ is a positive definite continuous function with decay at infinity. The KPP front speed formula is (see (4.2.21) in [22]):

$$
c^{*}=\inf _{\lambda_{2}>0} \frac{H\left(-\lambda_{1},-\lambda_{2}\right)}{\lambda_{2}},
$$

where:

$$
H(\lambda)=f^{\prime}(0)+\lim _{t \rightarrow \infty} t^{-1} \log E\left[\exp \left\{\lambda \cdot Y_{t}\right\}\right]
$$

and $Y_{t}$ is a diffusion process obeying the Ito equation:

$$
d Y_{s}=d W_{s}+\left(0, \sigma \xi\left(Y_{1, s}, t-s\right)\right) d s, \quad Y_{0}=0,
$$

$W_{s}$ is the standard two dimensional Wiener process.

It follows from (2.4) that:

$$
\begin{aligned}
Y_{1, t} & =W_{1, t}, \\
d Y_{2, s} & =d W_{2, s}+\sigma \xi\left(W_{1, s}, t-s\right) d s .
\end{aligned}
$$

So:

$$
E\left[e^{\lambda \cdot Y_{t}}\right]=E\left[e^{\lambda_{1} W_{1, t}+\lambda_{2} \sigma \int_{0}^{t} \xi\left(W_{1, s}, t-s\right) d s}\right] \cdot E\left[e^{\lambda_{2} W_{2, t}}\right] .
$$

The second factor equals $\exp \left\{\frac{\lambda_{2}^{2} t}{2}\right\}$. The first factor equals $u(0, t)$, with $u=u(x, t)$ the solution of:

$$
\begin{aligned}
& u_{t}=\frac{1}{2} u_{x x}+\lambda_{2} \sigma \xi(x, t) u, x \in R^{1}, \\
& u(x, 0)=e^{\lambda_{1} x}
\end{aligned}
$$

The solutions of (2.6) are invariant in the sense of distribution if $\left(\lambda_{1}, \lambda_{2}\right) \rightarrow-\left(\lambda_{1}, \lambda_{2}\right)$, $x \rightarrow-x$, implying that the $\operatorname{limit}: \lim _{t \rightarrow \infty} t^{-1} \log u(0, t) \equiv \gamma(\lambda)$ is an even function in $\lambda$, if it exists and is nonrandom.

The function $v=\log u(x, t)$ satisfies the viscous Hamilton-Jacobi equation:

$$
v_{t}=\frac{1}{2} v_{x x}+\frac{1}{2} v_{x}^{2}+\lambda_{2} \sigma \xi(x, t),
$$

with linear initial data $v(x, 0)=\lambda_{1} x$. The limit $\lim _{t \rightarrow \infty} t^{-1} v$ agrees with the homogenized Hamiltonian of the related homogenization problem, see [22] for more exposition of this connection. A useful consequence is that $\gamma$ is a convex function in $\lambda_{1}$ being the homogenization limit of a quadratic Hamiltonian with oscillating potential (for fixed $\lambda_{2}$ ), as seen in the effective Hamiltonian formula (4.2.20), section 4.2 of [22]. Results on homogenizing inviscid Hamilton-Jacobi equation with convex Hamiltonian and bounded random potentials can be found in [18], [19], [20]. 
Evenness and convexity of $\gamma$ in $\lambda_{1}$ implies that $\gamma\left(0, \lambda_{2}\right) \leq \gamma\left(\lambda_{1}, \lambda_{2}\right)$, and:

$$
c^{*}=\inf _{\lambda_{2}>0} \frac{H\left(0, \lambda_{2}\right)}{\lambda_{2}} .
$$

As $\lambda_{1}=0$, the problem reduces to the parabolic Anderson model, studied by Carmona, Molchanov, and coworkers, [4], [5], [6]. The limit,

$$
\lim _{t \rightarrow \infty} t^{-1} \log u(0, t)=\gamma
$$

if it exists and is nonrandom, is called the almost sure Lyapunov exponent.

A case where the limit $\gamma$ exists as a finite nonrandom number is when $\xi$ is white in time, or $\Gamma\left(t, y_{1}\right)=\delta(t) \Gamma_{0}\left(\left|y_{1}\right|\right)$. The spatially discrete case on lattice $Z^{d}$ was completely analyzed in [4], [6], where the Laplacian operator $\Delta_{x} v$ is replaced by $\sum_{\left|x^{\prime}-x\right|=1}\left(v\left(x^{\prime}\right)-v(x)\right)$. The main result is: Let $v$ be the solution of

$$
v_{t}=\kappa \Delta v+\xi(x, t) v, \quad x \in Z^{d}, \quad \kappa>0,
$$

with product in the Stratonovich sense, then the almost sure Lyapunov exponent $\gamma(\kappa)$ exists as nonrandom number and obeys $\left(b_{0}>0, d=1,2\right)$ :

$$
\begin{aligned}
& \gamma(\kappa) \sim \frac{b_{0}}{-\log \kappa}, \quad \kappa \ll 1, \\
& \gamma(\kappa) \sim \frac{\Gamma_{0}(0)}{2}, \quad \kappa \gg 1 .
\end{aligned}
$$

In three and higher dimensions, $\gamma(\kappa)=\Gamma_{0}(0) / 2$ if $\kappa$ exceeds a critical level $\kappa_{c r}$. This shows that the larger the diffusion effect, the more $\gamma$ tends to be constant $\Gamma_{0}(0) / 2$. Moreover, $\gamma(\kappa)$ is a monotone increasing continuous function in $\kappa$ [4]. The continuum case is more involved, Cranston and Montford just proved [8] that $\gamma(\kappa) \sim c_{1} \kappa^{p}$, $p \in(0,1 / 2), c_{1}>0, \kappa \ll 1$, and that $\gamma(\kappa)>0$ for all $\kappa>0$. The large $\kappa$ regime (2.11) is expected to be the same, also $\gamma(\kappa)$ is monotone in $\kappa$. If the continuum $\Delta$ is approximated by standard central differencing, we see that the continuum $\Delta$ is close to a discrete one with larger diffusion.

The results can be adapted to equation (2.6) with $\lambda_{1}=0$. First $\frac{1}{\sqrt{a}} \xi(y, t / a)=\xi(y, t)$ in law, for any $a>0$. Let $t=t^{\prime} / a, a=\left(\sigma \lambda_{2}\right)^{2}$, equation (2.6) becomes:

$$
u_{t^{\prime}}=\frac{1}{2 \sigma^{2} \lambda_{2}^{2}} \Delta u+\xi\left(t^{\prime}, x\right) u
$$

It follows that $\lim _{t \rightarrow \infty} u(0, t) / t=\gamma^{*}=\gamma^{*}\left(\sigma \lambda_{2}\right)$ such that

$$
\begin{aligned}
& \gamma^{*}\left(\sigma \lambda_{2}\right) \sim \frac{\Gamma(0)\left(\sigma \lambda_{2}\right)^{2}}{2}, \quad \sigma \lambda_{2} \ll 1, \\
& \gamma^{*}\left(\sigma \lambda_{2}\right) \sim b_{0} h\left(\frac{1}{2 \sigma^{2} \lambda_{2}^{2}}\right)\left(\sigma \lambda_{2}\right)^{2}, \quad \sigma \lambda_{2} \gg 1,
\end{aligned}
$$

where $h=h(x)=x^{p}, p \in(0,1 / 2)$. Now we minimize $H\left(0, \lambda_{2}\right) / \lambda_{2}$.

For $\sigma \ll 1$, the correction on the $H$ function from the shear field is small, so the minimizer is located at $\lambda_{2} \sim O(1)$, and we have:

$$
H\left(0, \lambda_{2}\right) / \lambda_{2} \sim f^{\prime}(0) / \lambda_{2}+\lambda_{2} / 2+\frac{\Gamma(0) \sigma^{2} \lambda_{2}}{2}+\text { h.o.t. }
$$


giving minimal point:

$$
\lambda_{2} \sim \sqrt{\frac{2 f^{\prime}(0)}{1+\Gamma(0) \sigma^{2}}},
$$

and minimum:

$$
c^{*}=\sqrt{2 f^{\prime}(0)}\left(1+\frac{1}{2} \Gamma(0) \sigma^{2}\right)+\text { h.o.t. }
$$

This is the stochastic analogue of quadratic speed enhancement.

For $\sigma \gg 1$, and $\lambda_{2} \sigma \gg 1$, we have:

$$
H\left(0, \lambda_{2}\right) / \lambda_{2} \sim b_{0} h\left(\frac{1}{2 \sigma^{2} \lambda_{2}^{2}}\right) \sigma^{2} \lambda_{2}+f^{\prime}(0) / \lambda_{2}+\lambda_{2} / 2,
$$

giving an upper bound with $\lambda_{2}=\frac{\sigma_{0}}{\sigma}, \sigma_{0}$ large enough constant so that $\sigma \gg \sigma_{0} \gg 1$ :

$$
c^{*}=\inf _{\lambda_{2}>0} H\left(0, \lambda_{2}\right) / \lambda_{2} \leq O\left(\sigma_{0}^{1-2 p} \sigma\right),
$$

which shows that the upper bound on the speed is linear in $\sigma \gg 1$.

To establish a similar lower bound, let us note that $\gamma(\kappa)$ is bounded away from zero by an amount $\gamma_{0}$ if $\kappa \geq \kappa_{0}$, and asymptotics $\gamma(\kappa) \sim c_{1} \kappa^{p}$ holds, for some $p \in(0,1 / 2)$, if $\kappa<\kappa_{0}$.

Now we estimate $c^{*}$ from below. If $2\left(\sigma \lambda_{2}\right)^{2} \leq \kappa_{0}^{-1}$, then:

$$
H\left(0, \lambda_{2}\right) / \lambda_{2} \geq \gamma_{0} \sigma^{2} \lambda_{2}+f^{\prime}(0) / \lambda_{2}+\lambda_{2} / 2 \equiv F\left(\lambda_{2}\right) .
$$

The absolute minimal point $\lambda_{2, m}$ of $F$ satisfies:

$$
1 / 2-f^{\prime}(0) / \lambda_{2}^{2}+\gamma_{0} \sigma^{2}=0,
$$

or:

$$
\lambda_{2, m}=\sqrt{\frac{2 f^{\prime}(0)}{1+2 \gamma_{0} \sigma^{2}}} \sim O\left(\sigma^{-1}\right), \quad \sigma \gg 1 .
$$

So $\lambda_{2, m} \sigma=O(1)$, and $H\left(0, \lambda_{2}\right) / \lambda_{2} \geq F\left(\lambda_{2}\right) \geq O(\sigma)$.

On the other hand, if $2\left(\sigma \lambda_{2}\right)^{2} \geq \kappa_{0}^{-1}$,

$$
H\left(0, \lambda_{2}\right) / \lambda_{2} \geq c_{1} 2^{-p} \sigma^{2-2 p} \lambda_{2}^{1-2 p}+f^{\prime}(0) / \lambda_{2}+\lambda_{2} / 2 \equiv G\left(\lambda_{2}\right) .
$$

The absolute minimal point $\lambda_{2, M}$ of $G$ obeys:

$$
c_{1} 2^{-p}(1-2 p) \sigma^{2-2 p} \lambda_{2}^{-2 p}+1 / 2=f^{\prime}(0) / \lambda_{2}^{2},
$$

implying:

$$
c_{1} 2^{-p}(1-2 p) \sigma^{2-2 p} \lambda_{2, M}^{-2 p} \leq f^{\prime}(0) / \lambda_{2, M}^{2},
$$

or:

$$
\lambda_{2, M} \leq C\left(p, c_{1}, f^{\prime}(0)\right) / \sigma,
$$


for some positive constant $C\left(p, c_{1}, f^{\prime}(0)\right)$. It follows from (2.20) and (2.19) that:

$$
H\left(0, \lambda_{2}\right) / \lambda_{2} \geq f^{\prime}(0) / \lambda_{2, M} \geq C^{\prime}\left(p, c_{1}, f^{\prime}(0)\right) \sigma .
$$

The above two lower bounds combine to give $c^{*} \geq O(\sigma)$, and together with upper bound (2.16), we have the front speed bound $c^{*}=O(\sigma)$ for large $\sigma$.

The $\gamma$ diverges logarithmically when $\xi=\xi(x)$ is a spatial Gaussian process [6]. The leading order exponential growth rate of $u$ depends on the fact that:

$$
\sup _{|x| \leq t} \xi(x) \sim \sqrt{2 \Gamma(0) \log t}, \quad t \rightarrow \infty, \text { a.s. }
$$

and so:

$$
\lim _{t \rightarrow \infty} \frac{1}{t \sqrt{\log t}} \log u(t, x)=\sigma\left|\lambda_{2}\right| \sqrt{2 \Gamma(0)}, \text { a.s. }
$$

implying front speed divergence in time. In other words, at large $t$ :

$$
\frac{H\left(0, \lambda_{2}\right)}{\lambda_{2}} \sim \frac{f^{\prime}(0)}{\lambda_{2}}+\frac{\lambda_{2}}{2}+\sigma \operatorname{sign}\left(\lambda_{2}\right) \sqrt{2 \Gamma(0) \log t},
$$

or:

$$
c^{*}=\inf _{\lambda_{2}>0} \frac{H\left(0, \lambda_{2}\right)}{\lambda_{2}} \sim c_{0}+\sigma \sqrt{2 \Gamma(0) \log t} .
$$

In view of (2.21), such divergence can be avoided if the front is restricted inside an infinite channel of finite cross section. However, the almost sure constant speed ceases to exist, as on a finite interval $\xi$ process loses ergodicity, different realizations can be either more positive or negative, so ensemble averaged speed becomes an alternative measure of front speed. A numerical study of random fronts in channels will be reported elsewhere [16].

3. Conclusions. We found that KPP front speeds are finite and enhanced through white in time stationary Gaussian random shears. The enhancement is quadratic in the root mean square (rms) of small shear amplitudes, and grows at the order of the large rms amplitudes. The front speeds diverge logarithmically in time independent mean zero Gaussian shears. The findings will help to investigate similar front behaviors under other forms of nonlinearities, and serve as a useful guide for numerical works on fronts through random shears in channels of large but finite cross sections.

Acknowledgements. The work was partially done during my visit to Inst $\mathrm{H}$. Poincaré (IHP) in Oct, 2002. I would like to thank H. Berestycki and J-M Roquejoffre for inviting and their hospitalities, as well as IHP for a visiting professorship. I also thank S. Molchanov and M. Cranston for communicating ongoing work on parabolic Anderson models. Partial NSF support (ITR-0219004) is gratefully acknowledged.

\section{REFERENCES}

[1] B. Audoly, H. Berestycki, Y. Pomeau, Raction-Diffusion en ećoulement rapide, Note C. R. Acad. Sc. Paris 328, Série II, 2000, pp. 255-262.

[2] A-L Barabási, H. E. Stanley, Fractal Concepts in Surface Growth, Cambridge University Press, 1995 
[3] H. BERESTYCKI, The influence of advection on the propagation of fronts in reaction-diffusion equations, Proceedings NATO ASI Conf. Cargese, H. Berestycki and Y. Pomeau eds, Kluwer, to appear.

[4] R. Carmona, S. Molchanov, Parabolic Anderson Model and Intermittency, Memoirs of the AMS, 108:518 (1994).

[5] R. Carmona, S. Molchanov, Stationary parabolic Anderson model and intermittency, Prob. Theory Rel. Fields 102 (1995), pp. 433-453.

[6] R. Carmona, L. Koralov, S. Molchanov, Asymptotics for the almost sure Lyapunov exponent for the solution of the parabolic Anderson problem, Random Oper. and Stoch. Equ., 9:1 (2001), pp. 77-86.

[7] P. Constantin, A. Kiselev, A. Oberman, L. Ryzhik, Bulk burning rate in passive-reactive diffusion, Arch Rat. Mech Analy, 154 (2000), pp. 53-91.

[8] M. CRAnston, personal communication, 2003.

[9] M. Freiduin, Functional Integration and Partial Differential Equations. Annals of Mathematics Studies, Number 109, Princeton University Press, 1985.

[10] S. HeINZE, The speed of travelling waves for convective reaction-diffusion equations, preprint 84, Max-Planck-Institut für Mathematik in den Naturewissenschaften, Leipzig, 2001.

[11] S. Heinze, G. Papanicolaou, A. Stevens, Variational principles for propagation speeds in inhomogeneous media, SIAM J. Applied Math, 62:1 (2001).

[12] A. KISEleV, L. RYZHiK, Enhancement of the traveling front speeds in reaction-diffusion equations with advection, Ann. de l'Inst. Henri Poincaré, Analyse Nonlinéaire, 18 (2001), pp. 309-358.

[13] A. Majda And P. Souganidis, Flame Fronts in a Turbulent Combustion Model with Fractal Velocity Fields, Comm Pure Appl Math, Vol. LI (1998), pp. 1337-1348.

[14] S. Molchanov, personal communication, 2002.

[15] J. Nolen, J. XIN, Analysis and computation of reaction-diffusion front speeds in spatiallytemporally periodic shear flows, SIAM Journal on Multiscale Modeling and Simulations, 2003, to appear.

[16] J. Nolen, J. XIN, A numerical study of reaction-diffusion front speeds in random shear flows, in preparation.

[17] G. Papanicolaou, J. Xin, Reaction-Diffusion Fronts in Periodically Layered Media, J. Stat. Physics, 63 (1991), pp. 915-931.

[18] F. Rezaknanlou, Central Limit Theorem for Stochastic Hamilton-Jacobi Equations, Comm. Math Physics, 211 (2000), pp. 413-438.

[19] F. Rezakhanlou, J. TARver, Homogenization for Stochastic Hamilton-Jacobi Equations, Arch. Rat. Mech. Anal., 151 (2000), pp. 277-309.

[20] P. E. Souganidis, Stochastic homogenization of Hamilton-Jacobi equations and some applications, Asymptotic Analysis, 20 (1999), pp. 1-11.

[21] N. Vladimirova, P. Constantin, A. Kiselev, O. Ruchaiskiy, L. Ryzhik, Flame Enhancement and Quenching in Fluid Flows, preprint, 2002.

[22] J. Xin, Front Propagation in Heterogeneous Media, SIAM Review, 42:2 (2000), pp. 161-230. 
J. XIN 